\title{
Management of Catastrophic Reaction in a Patient with Neurocognitive Disorder with Combination of Buspirone and Gabapentin- A Case Report
}

\author{
Ashish Sarangi* \\ Psychiatry Resident, Texas Tech Health Sciences Center, Lubbock, Texas, USA
}

*Corresponding author: Sarangi A, Psychiatry Resident, Texas Tech Health Sciences Center, Lubbock, Texas, USA, E-mail: ashish.sarangi@ttuhsc.edu

Received: 06 Dec, 2017 | Accepted: 30 Jan, 2018 | Published: 06 Feb, 2018

Citation: Sarangi A (2018) Management of Catastrophic Reaction in a Patient with Neurocognitive Disorder with Combination of Buspirone and Gabapentin- A Case Report. J Psychiatry Ment Health 3(1): dx.doi. org/10.16966/2474-7769.123

Copyright: (C) 2018 Sarangi A. This is an open-access article distributed under the terms of the Creative Commons Attribution License, which permits unrestricted use, distribution, and reproduction in any medium, provided the original author and source are credited.

\section{Introduction}

Catastrophic reactions (CR) have been well described in the literature as a state of agitation and helplessness that patients experience when they are challenged with tasks beyond their daily competency [1]. The prevalence of this condition is enough to warrant a closer analysis in the authors' opinion. In one study comprising of 146 patients with Alzheimer's disease, 16 percent were found to have a catastrophic reaction [2]. The literature however has not yet clearly identified a specific protocol in the management of this entity with specific pharmaceutical agents. Various classes of medications including SSRIs, anticonvulsants and atypical antipsychotics have been tried on an experimental basis with variably efficacy. Here I would like to present a case of SW who was managed successfully with a treatment combination of Buspirone and Gabapentin.

\section{Case Report}

A 63-year-old Caucasian woman with a previous diagnosis of depression and anxiety presented with a 2-day history of suicidal ideations with a plan to shoot herself with her husband's gun. She endorsed a significant depressive and anxiety symptom which was being managed by her primary outpatient psychiatrist. She was unsuccessfully tried on multiple psychotropic agents including Effexor, Lexapro, Trintellix and Seroquel. Along with the depressive and anxiety symptoms she also admitted to significant memory difficulties dating as far back as 14 years. Her memory impairments had progressed to the point that she would get lost while driving and needed occasional assistance with activities of daily living including flushing the toilet and putting on her clothes. A striking point about her presentation included repeated episodes of unprovoked crying without any obvious indication to do so. She was treated unsuccessfully for her neurocognitive issues with Aricept, Exelon path and Namenda.

On evaluation of the client during sessions, she was noted to have significant lability of her mood with frequent outbursts of crying without obvious stimuli. However, it was noted that her affect would significantly improve once she is engaged in conversation or in the presence of her family members during family meetings. Patient demonstrated significant deficits in her memory having no recollection initially of how she was brought to the hospital and identifying persons around her as being from a different state. Objective testing of her cognition however ranged from MOCA initially being 16 and then improving to 23 within a week's time and MMSE score of 23. She was diagnosed with Major Neurocognitive Disorder probable secondary to Alzheimer's Disease- severe with perceptual abnormalities.

The patient was commenced on a regime of Mirtazipine $15 \mathrm{mg}$ by mouth nightly which was titrated to a dose of $30 \mathrm{mg}$ by mouth nightly and Buspirone $5 \mathrm{mg}$ by mouth three times a day. She continued to endorse significant lability in her mood with alternating crying and laughing episodes within short term periods. MRI brain with and without IV contrast were obtained which showed mild microvascular change scattered in the subcortical and periventricular deep white matter bilaterally. B12, folate and thyroid studies obtained were normal. Over the course of the admission, Buspirone was increased to $10 \mathrm{mg}$ by mouth three times daily and Gabapentin was added at a dose of $100 \mathrm{mg}$ three times daily. Upon addition of Gabapentin notable improvement was seen in overall mood, affect and subjective anxiety. The patient's symptoms diminished within a matter of days of addition of Gabapentin and this was increased slowly to achieve maximal therapeutic response. Nursing staff reported decreased crying spells and the patient was able to maintain more sustained conversations. 


\section{Discussion and conclusion}

Aggressive behavior and mood lability has often been treated with low dose antipsychotics. However recent reports highlighting serious adverse effects of antipsychotic medications in the elderly population has brought to light the need for alternate agents in management of these patients [3]. Gabapentin is an anticonvulsant drug that mimics and potentiates GABAergic activity even though it does not bind to the same site as GABA [3]. An association between neurocognitive disorder, behavioral problems and GABA deficits may possibly explain the usefulness of Gabapentin in the treatment of CR [4]. A dosage range of $200 \mathrm{mg}$ orally up to a maximum dosage of $3600 \mathrm{mg}$ may be used in the authors' opinion based on clinical response and tolerability. Caution however needs to be maintained in using Gabapentin in the management of patients with Lewy Body Dementia where worsening of neuropsychiatric symptoms have been seen [5]. However, compared to antipsychotic medications, Gabapentin has shown to be safer in use for patients with cerebrovascular accidents. As a matter of fact, Gabapentin is the only anticonvulsant medication studied in the management of patients with stroke [6].

Gabapentin was used to previous limited evidence for mood lability and irritability in patients with dementia. Greater tolerability was also a factor in choice of Gabapentin as an agent for use.

Buspirone is an anxiolytic agent which is a partial 5- HT1A agonist. Although no studies have addressed the role of buspirone in combination with other agents in management of CR in patients with dementia, it demonstrated good efficacy for controlling breakthrough anxiety and mood lability in our index patient. Short term Buspirone treatment as monotherapy has shown evidence in the form of single case reports in the management of disinhibition in this patient population [7].

In conclusion, it should be noted that although neither buspirone nor gabapentin have been given approval by the FDA for management of $\mathrm{CR}$, they provide reasonable alternate options to management of such patients based on the provider's clinical judgement.

\section{Clinical pearls}

- Patients with dementia may present with a state of fluctuating agitation, lability and restlessness which is known as "catastrophic reaction"

- Catastrophic reaction may not improve with typical neurocognitive enhancers

- Add on Gabapentin may be of benefit in these patients due to increasing GABA activity and its neuroprotective effects

\section{Author Disclosure Information}

The author reports no potential conflict of interest in writing this report.

\section{References}

1. Yeom HA, Watson NM (2009) Patterns of antecedents of catastrophic reactions in nursing home residents with dementia in the United States. Asian Nurs Res (Korean Soc Nurs Sci) 3: 99110.

2. Tiberti C, Sabe L, Kuzis G, García Cuerva A, Leiguarda R, et al. (1998) Prevalence and correlates of the catastrophic reaction in Alzheimer's disease. Neurology 50: 546-548.

3. Cooney C, Murphy S, Tessema H, Freyne A (2013) Use of lowdose gabapentin for aggressive behavior in vascular and Mixed Vascular/Alzheimer Dementia. J Neuropsychiatry Clin Neurosci 25: $120-125$.

4. Hardy J, Cowburn R, Barton A, Reynolds G, Dodd P, et al. (1987) A disorder of cortical GABAergic innervation in Alzheimer's disease. Neurosci Lett 73: 192-196.

5. Rossi P, Serrao M, Pozzessere G (2002) Gabapentin-induced worsening of neuropsychiatric symptoms in dementia with lewy bodies: case reports. Eur Neurol 47: 56-57.

6. Silvestrelli G, Corea F, Micheli S, Lanari A (2010) Clinical pharmacology and vascular risk. Open Neurol J 4: 64-72.

7. Tiller JW, Dakis JA, Shaw JM (1988) Short-term buspirone treatment in disinhibition with dementia. Lancet 2: 510. 